\section{Spreadsheet software to assess the locomotor disability: Submitting the actual software}

Sir,

In a vast country like ours, we are often called upon as a part of our duty to assess disability resulting from trauma/ burns in our patients. The latest guideline issued by the Government of India, for the assessment of locomotor disability, appeared as a Gazette notification in June 2001. We use this guideline regularly in our unit. During routine use we found the calculations which form a part of the guideline to be time consuming and difficult to explain in the court.

With a goal to simplify the calculation, I have previously adapted the gazette notification to a spread sheet. ${ }^{[1]}$ In a spread sheet, the calculations happen automatically and they are reproducible in the form of a printout which can easily explain how the final figure was arrived at, in the court. Further, the spread sheet makes the whole process of assessment faster and user friendly. However, in the said article $^{[1]}$ although the utility of such software was presented and the software tested, it was not available for public use.

With the purpose of making this spread sheet software freely available (without any copy right) to all the care givers, who deal with trauma and burn patients and their rehabilitation,
I wish to submit the actual excel version (.xls) version of the file. I hereby submit two spread sheets, one for upper limb locomotor disability assessment and the other for the lower limb. Each of these programs was thoroughly tested for the entire range of values which are possible.

It may be noted that with the wide availability of apps which can run an .xls file in the android/iOS/windows operating systems, this spread sheet software can run on most mobile devices like the tablet and smart phone.

\section{Sunderraj Ellur}

Department of Plastic Surgery, St John's Medical College and Hospital, Bengaluru, Karnataka, India

Address for correspondence: Dr. Sunderraj Ellur, Department of Plastic Surgery, St. John's Medical College and Hospital, Sarjapura Road, Bengaluru, Karnataka, India. E-mail: sundernetra@gmail.com

\section{REFERENCE}

1. Ellur S. Spreadsheet software to assess locomotor disability to quantify permanent physical impairment. Indian J Plast Surg 2012;45:530-7.

\begin{tabular}{|l|l|}
\multicolumn{2}{c|}{ Access this article online } \\
\hline Quick Response Code: & Website: \\
\hline
\end{tabular}

\begin{tabular}{|c|c|c|c|c|c|c|c|c|c|c|}
\hline \multicolumn{11}{|c|}{$\begin{array}{c}\text { GUIDELINES FOR LOCOMOTOR DISABILITY ASSESSMENT TO QUANTIFY PERMANENT PHYSICAL IMPAIRMENT } \\
\text { AS PER THE GAZETTE OF INDIA NOTIFICATION } 2001\end{array}$} \\
\hline \multicolumn{11}{|c|}{ UPPER LIMB } \\
\hline \multicolumn{11}{|c|}{ (All Data entries to be made in GREY cells only / Enter power as a fraction, example: $3 / 5$ ) } \\
\hline \multicolumn{11}{|c|}{ DEPARTMENT OF PLASTIC, RECONSTRUCTIVE SURGERY\& BURNS. } \\
\hline \multicolumn{11}{|c|}{ INSTITUTION NAME } \\
\hline \multicolumn{11}{|c|}{ PATIENT NAME } \\
\hline \multicolumn{11}{|c|}{ AGE } \\
\hline \multicolumn{11}{|c|}{ SEX } \\
\hline \multicolumn{11}{|c|}{ OUT PATIENT NO } \\
\hline \multicolumn{11}{|c|}{ INPATIENT NO } \\
\hline \multicolumn{11}{|c|}{ DIAGNOSIS } \\
\hline \multirow{2}{*}{\multicolumn{2}{|c|}{ RANGE OF MOVEMENT(Active) }} & $\begin{array}{l}\text { NORMAL } \\
\text { VALUE } \\
\text { (DEGREES) }\end{array}$ & RIGHT & LEFT & $\begin{array}{l}\text { LOSS OF } \\
\% \text { RIGHT }\end{array}$ & $\begin{array}{l}\text { LOSS OF } \\
\% \text { LEFT }\end{array}$ & $\begin{array}{l}\text { MEAN } \\
\text { LOSS } \\
\text { RIGHT }\end{array}$ & $\begin{array}{l}\text { MEAN } \\
\text { LOSS } \\
\text { LEFT }\end{array}$ & $\begin{array}{l}\text { SUM OF } \% \\
\text { LOSS }{ }^{*} 0.3 \\
\text { RIGHT }\end{array}$ & $\begin{array}{c}\text { SUM OF } \% \\
\text { LOSS * } 0.3 \\
\text { LEFT }\end{array}$ \\
\hline & & \multicolumn{2}{|c|}{ RIGHT } & \multicolumn{2}{|c|}{0.0} & \multicolumn{3}{|c|}{ LEFT } & \multicolumn{2}{|c|}{0.0} \\
\hline \multirow[t]{3}{*}{ SHOULDER } & $\overline{F L X-E X T N}$ & 220 & 220 & 220 & 0.0 & $\overline{0.0}$ & \multirow{3}{*}{0.0} & \multirow{3}{*}{0.0} & \multirow{3}{*}{0.0} & \multirow{3}{*}{0.0} \\
\hline & ROTATION & 180 & 180 & 180 & 0.0 & 0.0 & & & & \\
\hline & $A B D-A D D$ & 180 & 180 & 180 & 0.0 & 0.0 & & & & \\
\hline \multirow[t]{2}{*}{ ELBOW } & FLX-EXTN & 150 & 150 & 150 & 0.0 & 0.0 & \multirow{2}{*}{0.0} & \multirow{2}{*}{0.0} & \multirow{2}{*}{0.0} & \multirow{2}{*}{0.0} \\
\hline & SUP-PRO & 180 & 180 & 180 & 0.0 & 0.0 & & & & \\
\hline \multirow[t]{2}{*}{ WRIST } & FLX-EXTN & 160 & 160 & 160 & 0.0 & 0.0 & \multirow{2}{*}{0.0} & \multirow{2}{*}{0.0} & \multirow{2}{*}{0.0} & \multirow{2}{*}{0.0} \\
\hline & RAD-ULNR D & 55 & 55 & 55 & 0.0 & 0.0 & & & & \\
\hline
\end{tabular}


Letter to Editor

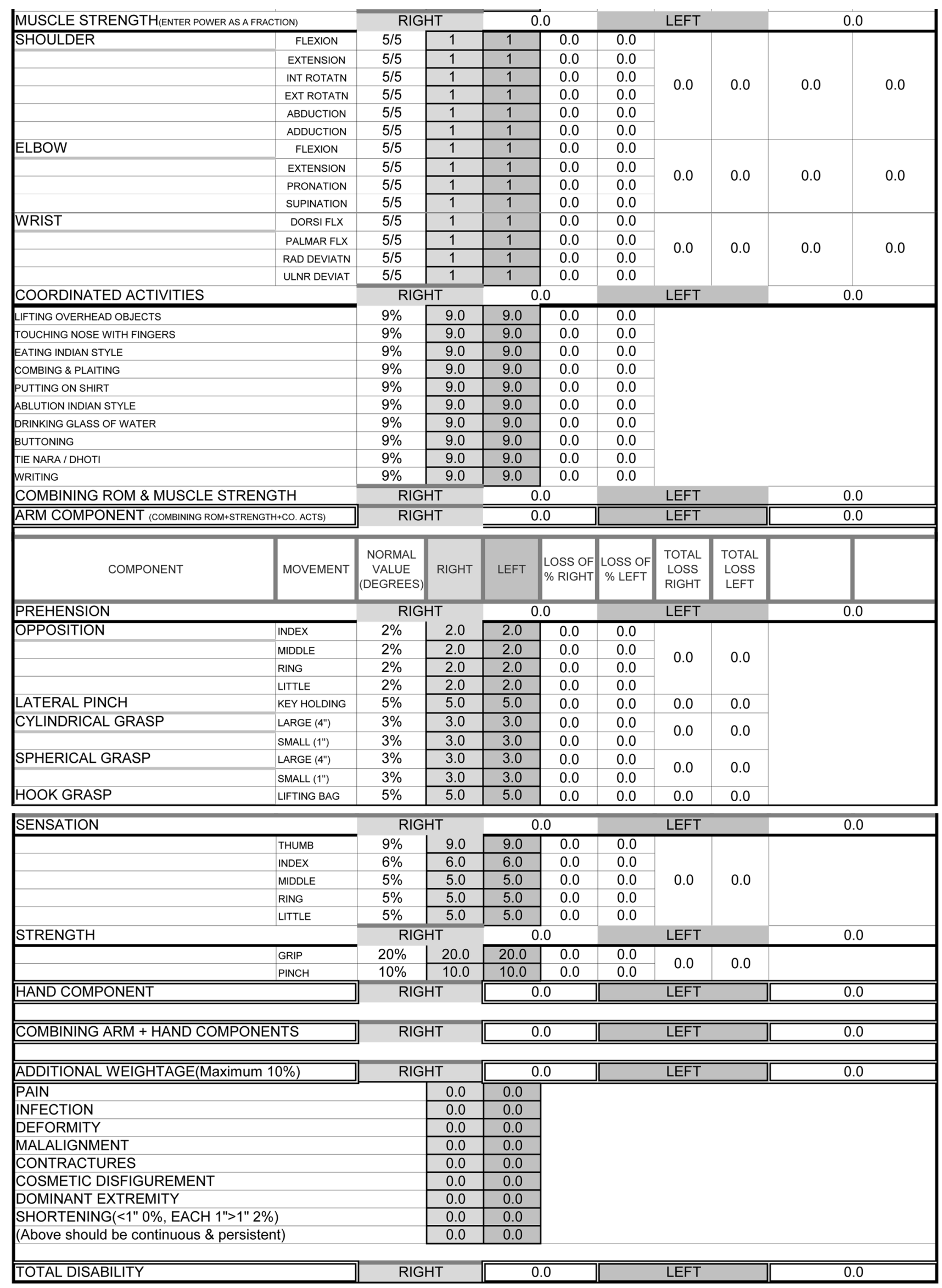


GUIDELINES FOR LOCOMOTOR DISABILITY ASSESSMENT TO QUANTIFY PERMANENT PHYSICAL IMPAIRMENT

\begin{tabular}{|c|c|c|c|c|c|c|c|c|c|c|}
\hline \multirow{2}{*}{\multicolumn{11}{|c|}{$\begin{array}{c}\text { GUIDELINES FOR LOCOMOTOR DISABILITY ASSESSMENT TO QUANTIFY PERMANENT PHYSICAL IMPAIRMENT } \\
\text { AS PER THE GAZETTE OF INDIA NOTIFICATION } 2001\end{array}$}} \\
\hline & & & & & & & & & & \\
\hline \multicolumn{11}{|c|}{ LOWER LIMB } \\
\hline \multirow{2}{*}{\multicolumn{11}{|c|}{$\begin{array}{c}\text { (All Data to be entered in GREY cells only / Enter Power as a fraction, example: } 3 / 5) \\
\text { DEPARTMENT OF PLASTIC, RECONSTRUCTIVE SURGERY\& BURNS. } \\
\end{array}$}} \\
\hline & & & & & & & & & & \\
\hline \multicolumn{11}{|c|}{ INSTITUTION NAME| } \\
\hline \multicolumn{11}{|c|}{ PATIENT NAME } \\
\hline \multirow{2}{*}{\multicolumn{11}{|c|}{ AGE }} \\
\hline \multirow{2}{*}{\multicolumn{11}{|c|}{$\begin{array}{r}\text { SEX } \\
\text { OUT PATIENT NO }\end{array}$}} \\
\hline & & & & & & & & & & \\
\hline \multicolumn{11}{|c|}{ INPATIENT NO } \\
\hline \multicolumn{11}{|c|}{ DIAGNOSIS| } \\
\hline COMPONENT & MOVEMENT & $\begin{array}{l}\text { NORMAL } \\
\text { VALUE } \\
\text { (DEGREES) }\end{array}$ & RIGHT & LEFT & $\begin{array}{l}\text { LOSS OF } \\
\% \text { RIGHT }\end{array}$ & $\begin{array}{l}\text { LOSS OF } \\
\% \text { LEFT }\end{array}$ & $\begin{array}{l}\text { MEAN LOSS } \\
\text { RIGHT }\end{array}$ & $\begin{array}{l}\text { MEAN LOSS } \\
\text { LEFT }\end{array}$ & $\begin{array}{l}\text { SUM OF \% } \\
\text { LOSS *0.3 } \\
\text { RIGHT }\end{array}$ & $\begin{array}{c}\text { SUM OF } \% \text { LOSS * } 0.3 \\
\text { LEFT }\end{array}$ \\
\hline \multicolumn{2}{|l|}{ RANGE OF MOVEMENT(Active) } & \multicolumn{2}{|c|}{ RIGHT } & \multicolumn{2}{|c|}{0.0} & & LEFT & & & 0.0 \\
\hline HIP & FLX-EXTN & 140 & 140 & 140 & 0.0 & 0.0 & & & & \\
\hline & ROTATION & 90 & 90 & 90 & 0.0 & 0.0 & 0.0 & 0.0 & 0.0 & 0.0 \\
\hline & $A B D-A D D$ & 90 & 90 & 90 & 0.0 & 0.0 & & & & \\
\hline KNEE & FLX-EXTN & 125 & 125 & 125 & 0.0 & 0.0 & 0.0 & 0.0 & 0.0 & 0.0 \\
\hline ANKLE \& FOOT & DORS-PLTR FLX & 70 & 70 & 70 & 0.0 & 0.0 & & 0 & ? & 0 \\
\hline & INV-EVERSION & 60 & 60 & 60 & 0.0 & 0.0 & 0.0 & 0.0 & 0.0 & 0.0 \\
\hline MUSCLE STRENGTH & TION) & RIG & & & .0 & & LEFT & & & 0.0 \\
\hline HIP & FLEXION & $5 / 5$ & 1 & 1 & 0.0 & 0.0 & & & & \\
\hline & EXTENSION & $5 / 5$ & 1 & 1 & 0.0 & 0.0 & & & & \\
\hline & INT ROTATN & $5 / 5$ & 1 & 1 & 0.0 & 0.0 & 00 & 00 & 00 & 00 \\
\hline & EXT ROTATN & $5 / 5$ & 1 & 1 & 0.0 & 0.0 & & 0.0 & 0.0 & 0.0 \\
\hline & ABDUCTION & $5 / 5$ & 1 & 1 & 0.0 & 0.0 & & & & \\
\hline & ADDUCTION & $5 / 5$ & 1 & 1 & 0.0 & 0.0 & & & & \\
\hline KNEE & FLEXION & $5 / 5$ & 1 & 1 & 0.0 & 0.0 & & & & \\
\hline & EXTENSION & $5 / 5$ & 1 & 1 & 0.0 & 0.0 & 0.0 & 0.0 & 0.0 & 0.0 \\
\hline ANKLE \& FOOT & DORSIFLX & $5 / 5$ & 1 & 1 & 0.0 & 0.0 & & & & \\
\hline & PLANTAR FLX & $5 / 5$ & 1 & 1 & 0.0 & 0.0 & & & & \\
\hline & INVERSION & $5 / 5$ & 1 & 1 & 0.0 & 0.0 & 0.0 & 0.0 & 0.0 & 0.0 \\
\hline & EVERSION & $5 / 5$ & 1 & 1 & 0.0 & 0.0 & & & & \\
\hline MOBILITY (COMBINING ROM \& MUSCLE STRENGTH) & & RIG & & & .0 & & LEFT & & & 0.0 \\
\hline COMPONENT & ATTRIBUTE & $\begin{array}{l}\text { NORMAL } \\
\text { VALUE } \\
\text { (DEGRES) }\end{array}$ & RIGHT & LEFT & $\begin{array}{l}\text { LOSS OF } \\
\% \text { RIGHT }\end{array}$ & $\begin{array}{l}\text { LOSS OF } \\
\% \text { LEFT }\end{array}$ & & & & \\
\hline STABILITY COMPONENT & & RIG & & & 0 & & LEFT & & & 0 \\
\hline WALKING ON PLAIN SURFACE & & 10 & 10 & 10 & 0 & 0 & & & & \\
\hline WALKING ON SLOPE & & 10 & 10 & 10 & 0 & 0 & & & & \\
\hline CLIMBING STAIRS & & 10 & 10 & 10 & 0 & 0 & & & & \\
\hline STANDING ON BOTH LEGS & & 10 & 10 & 10 & 0 & 0 & & & & \\
\hline STANDING ON AFFECTED LEG & & 10 & 10 & 10 & 0 & 0 & & & & \\
\hline SQUATTING ON FLOOR & & 10 & 10 & 10 & 0 & 0 & & & & \\
\hline SITTING CROSS LEG & & 10 & 10 & 10 & 0 & 0 & & & & \\
\hline KNEELING & & 10 & 10 & 10 & 0 & 0 & & & & \\
\hline TAKING TURNS & & 10 & 10 & 10 & 0 & 0 & & & & \\
\hline COMBINING MOBILITY \& STABILITY & & RIG & & & .0 & & LEFT & & & 0.0 \\
\hline EXTRA POINTS(MAXIMUM 10\%) & & RIG & & & 0 & & LEFT & & & 0 \\
\hline PAIN & & & & & & & & & & \\
\hline Severe & & 9 & & & & & & & & \\
\hline Moderate & & 6 & & & & & & & & \\
\hline Mild & & 3 & & & & & & & & \\
\hline DEFORMITY & & & & & & & & & & \\
\hline In Functional Position & & 3 & & & & & & & & \\
\hline In Non Functional Position & & 6 & & & & & & & & \\
\hline LOSS OF SENSATION & & & & & & & & & & \\
\hline Complete Loss & & 9 & & & & & & & & \\
\hline Partial Loss & & 6 & & & & & & & & \\
\hline COMPLICATIONS & & & & & & & & & & \\
\hline Superficial Complication & & 3 & & & & & & & & \\
\hline Deep Complication & & 6 & & & & & & & & \\
\hline SHORTENING & & & & & & & & & & \\
\hline First half inch & & 0 & & & & & & & & \\
\hline For every additional half inch & & 4 & & & & & & & & \\
\hline TOTAL DISABILITY & & RIG & & & $\overline{0.0}$ & & $\overline{\text { LEFT }}$ & & & $\overline{0.0}$ \\
\hline
\end{tabular}

